\title{
On a Fresnel-Integrals-Based Back-Scattering Model at Millimeter-Waves
}

\author{
Adrián Lahuerta-Lavieja*, Martin Johansson ${ }^{\dagger}$, Ulf Gustavsson ${ }^{\dagger}$, Guy A. E. Vandenbosch* \\ * ESAT-TELEMIC, KU Leuven, Leuven, Belgium \\ ${ }^{\dagger}$ Ericsson Research, Ericsson AB, Gothenburg, Sweden
}

\begin{abstract}
Geometry-based stochastic channel models (GSCMs) are suitable models at millimeter-wave (mm-wave) frequencies. Proper forward-scattering and back-scattering modeling is needed in order to realize realistic channel assessments. In this paper, a recently-proposed back-scattering model, 3D Fresnel, goes through further investigations: first, we investigate the impact of frequency in the results of a single scenario and, second, we study the effect of multiple surfaces. In both cases, the model shows good agreement with respect to the Physical Optics (PO) reference model.
\end{abstract}

\section{INTRODUCTION}

\section{A. Background}

Fifth-generation $(5 \mathrm{G})$ mobile communications systems are foreseen to make use of the high bandwidth availability at millimeter-wave (mm-wave) frequencies. Mm-wave frequencies are attractive since they only present greater losses in free space in comparison to the UHF band [1]. Besides, several mm-wave frequency bands present fairly small oxygenabsorption attenuation, e.g., 28, 38, and $60 \mathrm{GHz}$ [2].

Geometry-based stochastic channel models (GSCMs) have been proposed as one of the most suited channel models for millimeter wave frequencies [3]. GSCMs benefit from the advantages of geometry-based models, i.e., simplified ray tracing (RT), with the advantages of stochastic models (several carrier frequencies, empirical large and small scale parameters, etc.).

Blockage modeling is a common feature in GSCMs. A simple model based on multiple knife-edge diffraction (MKED) for calculating human body shadowing loss was proposed in [4], where rectangular screens are used to model the human body cross-section. This model was later on adopted by the METIS project [5, Sec. C.1.4]. A few years later, the mmMAGIC project extended this model to include phase variations around the edges of the blocking surface [6, Sec. 4.6.3]; a model which was adopted by ITU [7, Sec. 5.2.1.2]. Finally, Fresnel-integrals-based methods have also been proposed in literature for calculating body blockage phenomena [7, Sec. 5.2.1.1], [8]. All these models are based on exploiting the real scenario geometry, i.e., location, orientation, and size of the scattering objects and location and orientation of the antennas.

Inspired by these simple approaches based on rectangular surfaces, a method for calculating back-scattered fields from smooth rectangular surfaces was introduced in [9]. In fact, apart from proposing two new models based on the Fresnel integrals and the error function, the state-of-the-art forwardscattering models (METIS, ITU Fresnel and mmMAGIC/ITU $\mathrm{SE}$ ) were modified for capturing back-scattered fields as well.

\section{B. Paper Contributions}

In this paper, we present further tests to the 3D Fresnel back-scattering model. First, we investigate the model performance at different mm-wave frequencies in a unique scenario. Second, we test the model in a multiple-surface scenario. In addition, the results of both cases are validated using PO simulations.

\section{Paper Structure}

The rest of the paper is structured as follows. Section II describes the basis of the back-scattering model. Section III introduces the simulation settings, scenarios and results. Finally, Section IV concludes this paper.

\section{BRIEF DESCRIPTION OF THE MODEL}

The single-scattering method in [9] is fundamentally based on considering three steps: first, the propagation of the wave from the transmit antenna to the scatterer; second, the scattering at the finite scatterer; and third, the propagation of the wave from the scatterer to the Rx antenna.

The size and orientation of the scatterer is captured by a geometrical scattering coefficient $\Gamma_{i}^{\mathrm{G}}$ while the material properties are calculated in terms of Fresnel reflection coefficients $\Gamma_{i}^{\mathrm{F}}[10, \mathrm{pp} .179-180]$.

These scalar methods essentially evaluate the excess path length via the edges of the surface with respect to the length of the shortest path, i.e., the path via the specular reflection point on the plane defined by the scattering surface. Besides, the procedure includes antenna gains and is reciprocal. The methods are valid when the surface is visible from both antennas and the transmitter and receiver are on the same side of the plane defined by the surface. In addition, the GSCM structure is preserved by keeping the point-scatterer perspective to calculate the wave's phase delay.

Among the models in [9], the introduced 3D Fresnel model showed to be the most accurate - in terms of error metricscompared to a Physical Optics (PO) reference model when tested for different angles of incidence and departure, orientations, and distances with respect to the scattering surface. For this reason, we simply focus on the 3D Fresnel model in this paper. 


\section{RESULTS}

\section{A. Simulation Settings}

The main carrier frequency used here is $28 \mathrm{GHz}$, although simulations results at 39 and $60 \mathrm{GHz}$ are also presented. The antennas used in the simulations (unless otherwise stated) are models of the ones used in [11]: Schwarzbeck 9170 and Narda V637 for the transmit (Tx) and receive (Rx) antennas, respectively, scaled with the wavelength to have the same radiation properties at all frequencies. Finally, all scatterers are assumed to be perfect electric conductors (PEC), i.e., the Fresnel reflection coefficient $\Gamma^{\mathrm{F}}$ is -1 .

\section{B. Frequency Test}

For the test over frequency we will use Scenario A from [9]. This scenario consists of a $0.3 \times 0.5$-meter PEC rectangle which is rotated clockwise from 0 - the rectangle extending downwards as shown in the center of Fig. 1(a) - to 180 degrees around the rotation point $\mathrm{P}_{\mathrm{R}}$. The Tx and $\mathrm{Rx}$ antennas are collocated at one meter distance from the plane of the rectangle and pointed to the origin $\mathrm{O}$, i.e., normal incidence to the rectangle plane as shown in Fig. 1(a).

Amplitude and phase results of the 3D Fresnel model and $\mathrm{PO}$ at 28, 39 and $60 \mathrm{GHz}$ are shown in Figs. 1(b) and 1(c), respectively. Good agreement between the model and the PO reference both in amplitude and phase is shown for the three investigated frequencies, especially when the specular reflection point lies on the surface of the scatterer.

The main difference between the results of the three investigated frequencies is the power level reduction due to the decrease of the $\mathrm{Rx}$ antenna effective area as the frequency increases.

\section{Multiple-Surface Test}

Second, we define a scenario containing two $0.5 \times 0.5$-meter surfaces, see Fig. 2(a): Surface 1 is located closer to the Tx antenna at the starting position and moved along the $\mathrm{x}$-axis whereas Surface 2 is static and located with its centroid at the midpoint of the distance between the antennas along the $\mathrm{x}$-axis. In this example, Surface 1 is moved along the $\mathrm{x}$-axis in the $[-1,+1]$ meter interval while isotropic Tx and $\mathrm{Rx}$ antennas have been used to provide comparable power levels at the $\mathrm{Rx}$ antenna due to the direct and the scattered-from-the-rectangles components. Furthermore, only single-scattering (first-order) is considered both for the 3D Fresnel model and PO.

Amplitude and phase results of 3D Fresnel and PO are shown in Figs. 2(b) and 2(c), respectively. In addition, all the individual components have also been included in Figs. 2(b) and 2(c) to help the visualization of Surface 1 effect on this test. The 3D Fresnel model achieves very good performance with respect to the PO reference.

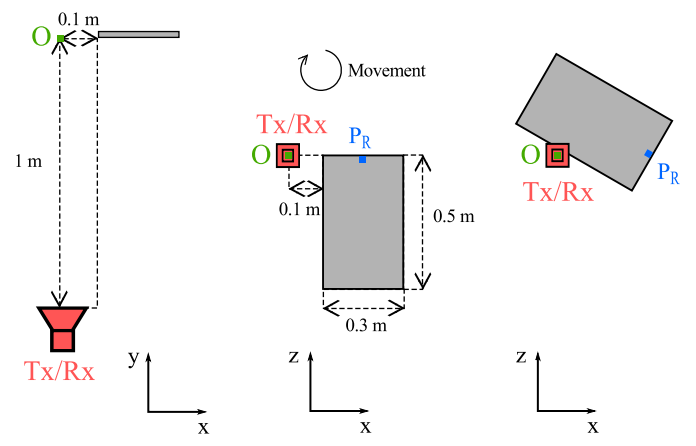

(a)

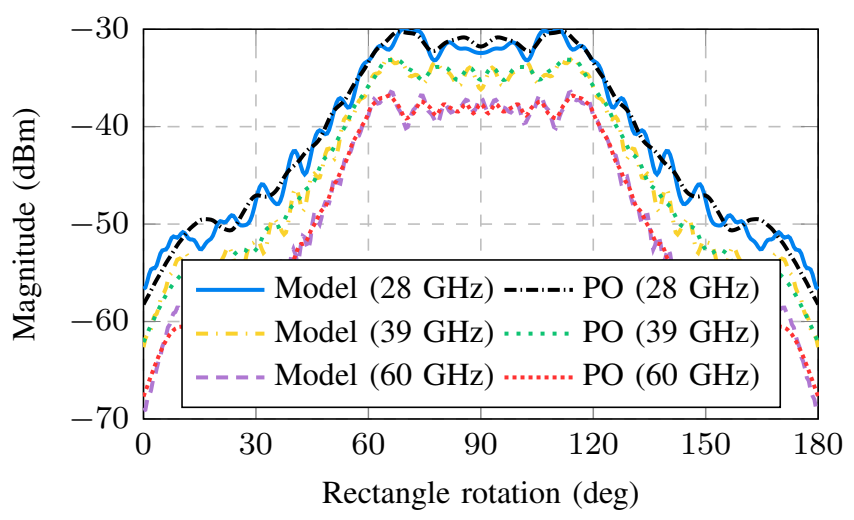

(b)

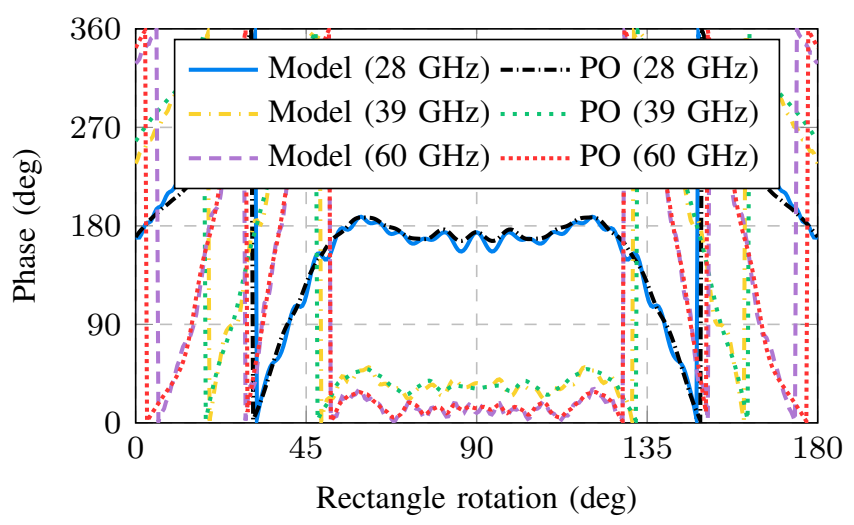

(c)

Fig. 1. Test case with of a normal-incidence rotating rectangle at 28, 39, and $60 \mathrm{GHz}$. (a) From left to right: top projection and side projection for 0and 120-degree rotation around $\mathrm{P}_{\mathrm{R}}$, respectively; (b) amplitude and (c) phase results for different rectangle rotations.

\section{CONCLUSION}

In this paper, performance of a 3D Fresnel back-scattering model at different mm-wave frequencies has been demonstrated. In addition, single-scattering from multiple surfaces has been shown.

\section{ACKNOWLEDGMENT}

This project has received funding from the European Union's Horizon 2020 research and innovation programme under the Marie Sklodowska-Curie grant agreement No. 721732. 

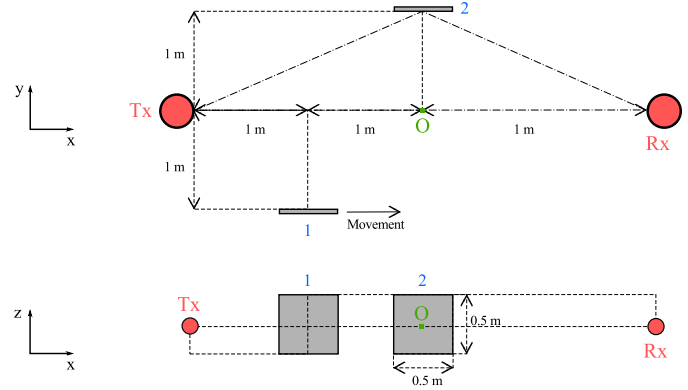

(a)

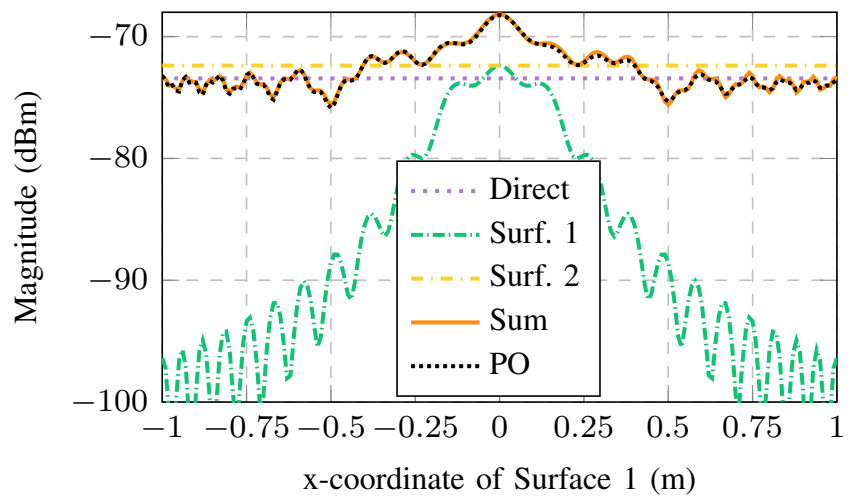

(b)

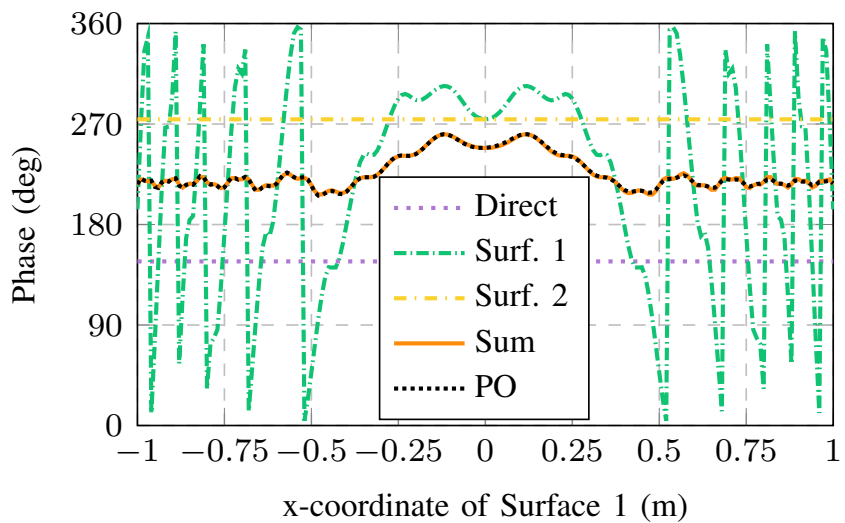

(c)

Fig. 2. Test-case with a moving surface (Surface 1) and a static surface (Surface 2). (a) From top to bottom: top projection and side projection, respectively, of the starting position of the moving surface; (b) amplitude and (c) phase results for different positions of Surface 1.

\section{REFERENCES}

[1] T. S. Rappaport, G. R. MacCartney, M. K. Samimi, and S. Sun, "Wideband millimeter-wave propagation measurements and channel models for future wireless communication system design," IEEE Trans. Commun., vol. 63, no. 9, pp. 3029-3056, Sep. 2015.

[2] T. S. Rappaport et al., "Millimeter wave mobile communications for 5G cellular: It will work!" IEEE Access, vol. 1, pp. 335-349, 2013.

[3] M. Shafi et al., "5G: A tutorial overview of standards, trials, challenges, deployment, and practice," IEEE J. Sel. Areas Commun., vol. 35, no. 6, pp. 1201-1221, Jun. 2017

[4] J. Medbo and F. Harrysson, "Channel modeling for the stationary UE scenario," in Proc. European Conf. Antennas Prop. (EuCAP), Apr. 2013, pp. 2811-2815.
[5] METIS, "METIS channel models," Mobile and Wireless Commun Enablers for the 2020 Inf. Soc. (METIS), Tech. Rep. D1.4 v3, Jul. 2015.

[6] mmMAGIC, "Measurement results and final mmMAGIC channel models," mm-Wave based Mobile Radio Access Netw. for 5G Integrated Commun. (mmMAGIC), Tech. Rep. D2.2 v2, May 2017.

[7] ITU-R, "Propagation by diffraction," Int. Telecommun. Union (ITU), Tech. Rep. P.526, Jan. 2018

[8] Z. Wang, H. Liu, S. Xu, X. Bu, and J. An, "A diffraction measurement model and particle filter tracking method for RSS-based DFL," IEEE J. Sel. Areas Commun., vol. 33, no. 11, pp. 2391-2403, Nov. 2015.

[9] A. Lahuerta-Lavieja, M. Johansson, U. Gustavsson, T. A. H. Bressner and G. A. E. Vandenbosch, "Computationally-efficient millimeter-wave back-scattering models," to be published in IEEE Trans. Antennas Propag., 2020.

[10] C. A. Balanis, Advanced Engineering Electromagnetics, 2nd ed. Hoboken, NJ: Wiley, 2012.

[11] A. Lahuerta-Lavieja, M. Johansson, G. A. E. Vandenbosch, and U Gustavsson, "Environment-embedded radiation patterns at millimeterwave frequencies," in Proc. European Conf. Antennas Prop. (EuCAP), Mar. 2019, pp. 1-5. 